ARTIGO ORIGINAL

\title{
Saturação por bases no crescimento e na qualidade de mudas de paricá
}

\author{
Bases saturation on growth and quality of parica seedlings \\ Cristiane Ramos Vieira ${ }^{1}$ (D), Oscarlina Lúcia dos Santos Weber ${ }^{2}$ (D), José Fernando Scaramuzza² (i) \\ ${ }^{1}$ Universidade de Cuiabá - UNIC, Cuiabá, MT, Brasil \\ ¿2Universidade Federal de Mato Grosso - UFMT, Cuiabá, MT, Brasil
}

Como citar: Vieira, C. R., Weber, O. L. S., \& Scaramuzza, J. F. (2020). Saturação por bases no crescimento e na qualidade de mudas de paricá. Scientia Forestalis, 48(125), e2934. https://doi.org/10.18671/scifor.v48n125.07

\begin{abstract}
Resumo
Schizolobium amazonicum (Huber ex Ducke) é uma espécie florestal cuja madeira possui qualidade para ser utilizada pela indústria moveleira, dessa forma, crescem as áreas de plantio com essa espécie. No entanto, faz-se necessário o conhecimento a respeito de suas exigências nutricionais ainda na fase de viveiro. Portanto, o estudo teve por objetivo verificar a influência da saturação por bases no crescimento e na qualidade de mudas de S. amazonicum. O delineamento experimental utilizado foi o inteiramente casualizado, com cinco tratamentos e cinco repetições: T0 - testemunha; T1 - 40\% de saturação por bases; T2 - 60\% de saturação por bases; T3 - 70\% de saturação por bases; T4 - 90\% de saturação por bases. Avaliando-se, após 120 dias: altura, diâmetro de coleto, relação entre altura e diâmetro; biomassa das partes aérea e radicular; relação entre biomassa na parte aérea e biomassa na parte radicular; relação entre altura e biomassa da parte aérea; índice de qualidade de Dickson e; a concentração de nutrientes na parte aérea das mudas. O crescimento das mudas de S. amazonicum foi pouco influenciado pela saturação por bases, no entanto, alterou os teores de macro e de micronutrientes, recomendando-se a saturação de 70\% para a espécie.
\end{abstract}

Palavras-chave: Schizolobium amazonicum; Calagem; Nutrição.

\begin{abstract}
Schizolobium amazonicum (Huber ex Ducke) is a forest species whose wood has quality to be used by the furniture industry, in this way, grow the areas of planting with it. However, it is necessary to know about their nutritional requirements at the nursery stage. The study aimed to assess the effect of cation saturation on growth and quality of S. amazonicum. The experimental design was completely randomized with five treatments: T0 - control; T1 - 40\% saturation; T2 - 60\% saturation; T3 - 70\% saturation; T4 - 90\% saturation. Were evaluated after 120 days: height, diameter, relation between height and diameter; shoot and roots biomass; relationship between height and aerial biomass; relationship between aerial biomass and roots biomass; Dickson quality index; and nutrients concentration in aerial part. The cation saturation did not influence the growth of S. amazonicum seedlings. The growth of S. amazonicum seedlings was little influenced by base saturation, however, it altered the macro and micronutrients contents, recommending the saturation of $70 \%$ for the species.
\end{abstract}

Keywords: Schizolobium amazonicum; Liming; Nutrition.

\section{INTRODUÇÃO}

Os solos sob Cerrado são, em geral, de elevada acidez; altos teores de alumínio trocável; baixa disponibilidade de cátions básicos como $\mathrm{Ca}, \mathrm{Mg}$ e do $\mathrm{P}$ na sua forma assimilável; características que comprometem o crescimento das plantas. Dessa maneira, anteriormente ao plantio, torna-se indispensável corrigir a fertilidade do solo.

Fonte de financiamento: Nenhuma.

Conflito de interesse: Nada a declarar.

Autor correspondente: cris00986@hotmail.com

Recebido: 14 junho 2018

Aceito: 9 maio 2019

Editor: Francides Gomes da Silva Júnior.

(c) (i) Este é um artigo publicado em acesso aberto (Open Access) sob a licença Creative Commons Attribution, que permite uso, distribuição e reprodução cc. em qualquer meio, sem restrições desde que o trabalho original seja corretamente citado. 
A técnica de correção mais utilizada é a calagem, que, neutraliza a acidez do solo, além de disponibilizar Ca e Mg e favorecer a do P, contribuindo para o aumento da fertilidade e, dessa forma, para a melhoria da nutrição das plantas. Sendo, o método mais utilizado para determinar a necessidade de correção da acidez, o da elevação da saturação por bases.

Segundo Oliveira et al. (2015) a adequação do pH e o equilíbrio entre os teores de bases no solo proporciona uma adequada disponibilidade da maioria dos nutrientes para as plantas. Além disso, a calagem também diminui a precipitação do $\mathrm{P}$ com o Al trocável, pois os grupos hidroxila $\left(\mathrm{OH}^{-}\right)$liberados complexam o $\mathrm{Al}^{3+}$ (Cardoso et al., 2015).

No entanto, deve-se ressaltar que cada espécie responde de uma forma à adição de calcário. Diante disso, desenvolveu-se experimento com a espécie florestal S. amazonicum, que pertence à família Caesalpiniaceae e é conhecida como paricá. Essa espécie se caracteriza pelo rápido crescimento, boa adaptabilidade e potencial de produção (Gazel Filho et al., 2007). Pode ser utilizada em plantios homogêneos ou consorciados (Iwakiri et al., 2010), recomposição de áreas degradadas e reflorestamento (Gazel Filho et al., 2007), na confecção de chapas aglomeradas (Colli et al., 2010), na construção civil (Almeida et al., 2013) e na produção de energia (Vidaurre et al., 2012).

Quanto às suas exigências nutricionais, Marques et al. (2004a) observaram que N e Fe foram, respectivamente, o macro e o micronutriente mais requeridos. Porém, são raras as informações sobre a adubação para a espécie (Caione et al., 2012). Portanto, torna-se importante pesquisar sobre as condições de saturação por bases mais adequadas ao crescimento do S. amazonicum.

Respostas variadas foram observadas por diversos autores, ao aplicar calcário no substrato utilizado para produção de mudas de diferentes espécies florestais. Oliveira et al. (2015) recomendaram a saturação de 60 a 75\% para a produção de mudas de Toona ciliata (M. Roem,). em Cambissolo háplico. Já Braga et al. (2015) recomendaram 50\% de saturação para a mesma espécie, porém, ao utilizar como substrato Latossolo Vermelho distroférrico típico.

Vargas \& Marques (2017) verificaram que a aplicação de calcário favoreceu o crescimento e a qualidade das mudas de Anadenanthera colubrina (Vell.) Brenan e de Senna multijuga (Rich.) H.S.Irwin \& Barneby, como efeito da neutralização da acidez do solo e da elevação dos teores de $\mathrm{Ca}^{2+}$ e $\mathrm{Mg}^{2+}$ no solo. Enquanto que, Cardoso et al. (2015) constataram que, a aplicação de $44 \mathrm{mg} \mathrm{dm}^{-3}$ de $\mathrm{P}$ associada à adição de $500 \mathrm{mg} \mathrm{dm}^{-3}$ de calcário dolomítico proporcionaram a máxima produção econômica de matéria seca total de mudas de Swietenia macrophylla King.

No entanto, Freitas et al. (2017) ao estudar os efeitos da adubação fosfatada e da saturação por bases na produção de mudas de Plathymenia foliolosa Benth. recomendaram $300 \mathrm{mg} \mathrm{dm}^{-3}$ de $\mathrm{P}$, porém, não observaram a necessidade da calagem para a espécie.

Dessa forma, o estudo teve por objetivo avaliar o crescimento inicial e a qualidade de mudas de S. amazonicum submetidas a diferentes níveis de saturações por bases.

\section{MATERIAL E MÉTODOS}

O experimento foi realizado em casa de vegetação, situada nas coordenadas 15 36'39,46"S e $56^{\circ} 3^{\prime}$ 53,96"O, pertencente à Faculdade de Agronomia e Zootecnia (FAAZ) da Universidade Federal de Mato Grosso (UFMT), em ambiente sem controle de temperatura e umidade, construída de material telado com sombrite lateral e coberta com telha de amianto.

As sementes de S. amazonicum foram coletadas no município de Alta Floresta - MT e, para a produção das mudas, passaram por processo de quebra de dormência utilizando o método de choque térmico. As sementes foram imersas em água quente seguida da imersão em água fria. Logo após, foram dispostas em canteiro de 1,20 m x 3,00 m preenchido com areia, a uma profundidade de um cm, recomendada por Rosa et al. (2009). Permanecendo a pleno sol, com irrigação duas vezes ao dia (manhã e tarde). As primeiras germinações foram observadas cinco dias após a semeadura e ocorreu de maneira uniforme. Transcorridos mais 20 dias, as mudas atingiram comprimento de $15 \mathrm{~cm}$, estando aptas ao transplante.

O solo utilizado foi o Latossolo Vermelho distrófico com textura franco arenosa (Empresa Brasileira de Pesquisa Agropecuária, 2006), coletado em área de Cerrado nativo do Instituto Federal de Mato Grosso, campus de São Vicente da Serra. Uma amostra do solo foi seca ao ar, peneirada em malha de $2 \mathrm{~mm}$ e submetida à caracterização química seguindo métodos da 
Embrapa (Empresa Brasileira de Pesquisa Agropecuária, 1997) (Tabela 1). Em seguida, o solo foi misturado ao corretivo para a elevação da saturação por bases e deixado para incubação por 20 dias, seguindo a Equação 1. Como corretivo foi utilizado calcário com PRNT de 100\%, com óxido de cálcio e óxido de magnésio na relação 1:1,5.

$N C\left({ }_{t / h a}\right)=\left(V_{E}-V_{A}\right) T / 100$

Onde: $N C=$ necessidade de calagem em toneladas por hectare; $V_{E}=$ saturação por bases

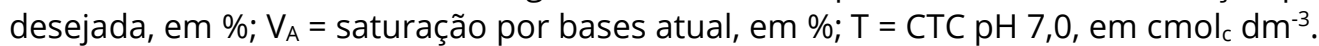

Tabela 1. Características químicas iniciais do solo

\begin{tabular}{|c|c|c|c|c|c|c|c|c|c|c|c|}
\hline pH & $\mathrm{H}+\mathrm{Al}$ & Al & $\mathrm{Ca}^{2+}$ & $\mathrm{Mg}^{2+}$ & K & $\mathbf{P}$ & SB & $T(p H 7,0)$ & $\mathbf{t}$ & v & m \\
\hline $\mathrm{CaCl}_{2}$ & \multicolumn{4}{|c|}{$\mathrm{cmol}_{\mathrm{c} \cdot} \cdot \mathrm{dm}^{-3}$} & \multicolumn{2}{|c|}{$\mathrm{mg} \mathrm{dm}^{-3}$} & \multicolumn{3}{|c|}{$\mathrm{cmol}_{\mathrm{c} \cdot} \cdot \mathrm{dm}^{-3}$} & \multicolumn{2}{|c|}{$\%$} \\
\hline 4,39 & 4,22 & 1,03 & 1,0 & 0,5 & 13,56 & 13,9 & 1,53 & 5,75 & 2,56 & 26,5 & 40,2 \\
\hline
\end{tabular}

pH em CaCl - relação 1:2,5; H+Al - em acetato de cálcio; $\mathrm{Al}_{1} \mathrm{Ca}^{2+}$ e $\mathrm{Mg}^{2+}$ - em KCl 1N; P e K - em Mehlich; SB - soma de bases; T (pH7,0) - capacidade de troca de cátions a pH 7,0; t efetiva - CTC efetiva; V\% - saturação por bases, em \%; m\% - saturação por Al, em \%.

O delineamento utilizado foi o inteiramente casualizado, com cinco tratamentos e cinco repetições: testemunha (solo de mata nativa, $V=26 \%$ ); $V=40 \% ; V=60 \%, V=70 \%$ e $V=90 \%$. O solo calcareado recebeu adubação básica, segundo Passos (1994), com $100 \mathrm{mg} \mathrm{dm}^{-3} \mathrm{de} \mathrm{N}$, $300 \mathrm{mg} \mathrm{dm}^{-3}$ de $\mathrm{P}, 100 \mathrm{mg} \mathrm{dm}^{-3}$ de $\mathrm{K}$ e $40 \mathrm{mg} \mathrm{dm}^{-3}$ de $\mathrm{S}$, nas fontes $\mathrm{NH}_{4} \mathrm{NO}_{3}, \mathrm{KH}_{2} \mathrm{PO}_{4}, \mathrm{KCl}$ e $\mathrm{K}_{2} \mathrm{SO}_{4}$, respectivamente. A solução de micronutrientes foi composta por $0,81 \mathrm{mg} \mathrm{dm}^{-3} \mathrm{de} \mathrm{B}_{\text {, }}$ $3,66 \mathrm{mg} \mathrm{dm}^{-3}$ de $\mathrm{Mn}, 4,0 \mathrm{mg} \mathrm{dm}^{-3}$ de $\mathrm{Zn}, 1,33 \mathrm{mg} \mathrm{dm}^{-3}$ de $\mathrm{Cu}$ e $0,15 \mathrm{mg} \mathrm{dm}^{-3}$ de $\mathrm{Mo}$, respectivamente, nas fontes $\mathrm{H}_{3} \mathrm{BO}_{3}, \mathrm{MnCl}_{2} .4 \mathrm{H}_{2} \mathrm{O}, \mathrm{ZnSO}_{4} .7 \mathrm{H}_{2} \mathrm{O}, \quad \mathrm{CuSO}_{4} .5 \mathrm{H}_{2} \mathrm{O}$ e $\left(\mathrm{NH}_{4}\right)_{6} \mathrm{Mo}_{7} \mathrm{O}_{24} .4 \mathrm{H}_{2} \mathrm{O}$, segundo Alvarez (1974).

Após a adubação básica, o solo foi utilizado para preencher os sacos plásticos de polipropileno sanfonado de $20 \times 30 \mathrm{~cm}$ com furos na parte inferior, considerando os respectivos tratamentos, em seguida, as mudas foram transplantadas para os mesmos. A adubação de cobertura foi realizada com adubações parceladas de $\mathrm{N}$ aos 40, 70 e 100 dias após o transplante, considerando a dose da adubação básica e como fonte o $\mathrm{NH}_{4} \mathrm{NO}_{3}$ P.A.

Os parâmetros morfológicos analisados para caracterizar o crescimento das mudas, aos 120 dias, foram: altura da parte aérea $(\mathrm{H})$, medida com régua graduada, desde a superfície do solo até as últimas folhas e diâmetro de colo (DC), medido com paquímetro digital. Em seguida, as cinco mudas de cada tratamento foram seccionadas em folhas, caule e raiz, secas em estufa de circulação forçada de ar e pesadas em balança analítica com precisão de 0,0005g, para determinações da biomassa seca; relação entre biomassa da parte aérea e biomassa da parte radicular; relação entre altura e biomassa da parte aérea. Após essas determinações aplicou-se o índice de qualidade de Dickson (Dickson et al., 1960).

O material vegetal seco (das folhas) foi moído e, submetido às digestões nitro-perclórica e sulfúrica segundo metodologia de Malavolta et al. (1997). A saber: $N$ total por semi-micro Kjeldahl; P por colorimetria do metavanadato; S por turbidimetria do sulfato de bário; K por fotometria de chama de emissão; Ca e Mg por quelatometria com EDTA; B por colorimetria da azometina $\mathrm{He}$ e; $\mathrm{Cu}, \mathrm{Fe}, \mathrm{Mn}$, Zn por espectrofotometria de absorção atômica.

Para o processamento e análises dos dados utilizou-se o Assistat 7.7 beta, e a análise estatística foi realizada aplicando-se a técnica de análise de variância e comparação múltipla de médias pelo método de Tukey a $5 \%$. Também foi utilizada a análise de regressão na definição de equações e posterior ajuste em planilha do Excel, para cada parâmetro avaliado. Para o cálculo da máxima eficiência técnica (MET) de cada variável utilizou-se a metodologia de Stork et al. (2000), a partir da Equação 2: 
$M E T=-b 1 / 2 b 2$

Onde: $X$ = ponto de máxima eficiência técnica; b1 e b2 = coeficientes da equação.

Para o cálculo da máxima eficiência econômica (MEE), utilizou-se a Equação 3:

$M E E=((t / w)-b 1)) / 2 b 2$

Onde: $\mathrm{w}=$ como o preço do produto, que foi de $\mathrm{R} \$ 40,00$ correspondente ao preço do saco de um quilo de calcário, em média; e $\mathrm{t}$ = correspondente ao preço do produto no qual utilizou-se o preço da muda de $S$. amazonicum, que está na média de $\mathrm{R} \$ 0,80$.

\section{RESULTADOS E DISCUSSÃO}

\section{Crescimento das mudas}

O crescimento das mudas de S. amazonicum foi pouco influenciado pela elevação da saturação por bases (Entra Figura 1), não se observando significância para os crescimentos em diâmetro, biomassa da parte radicular e para o índice de qualidade de Dickson. Isso pode ser atribuído à saturação por bases inicial (26\%), que pode ter propiciado condições adequadas ao crescimento das mudas e às exigências nutricionais da espécie que podem ter sido atendidas pelas características iniciais do solo utilizado no experimento. Cruz et al. (2004), Gomes et al. (2004) e Souza et al. (2008) também observaram pouca ou nenhuma resposta à adição de corretivo na produção de mudas de espécies nativas.
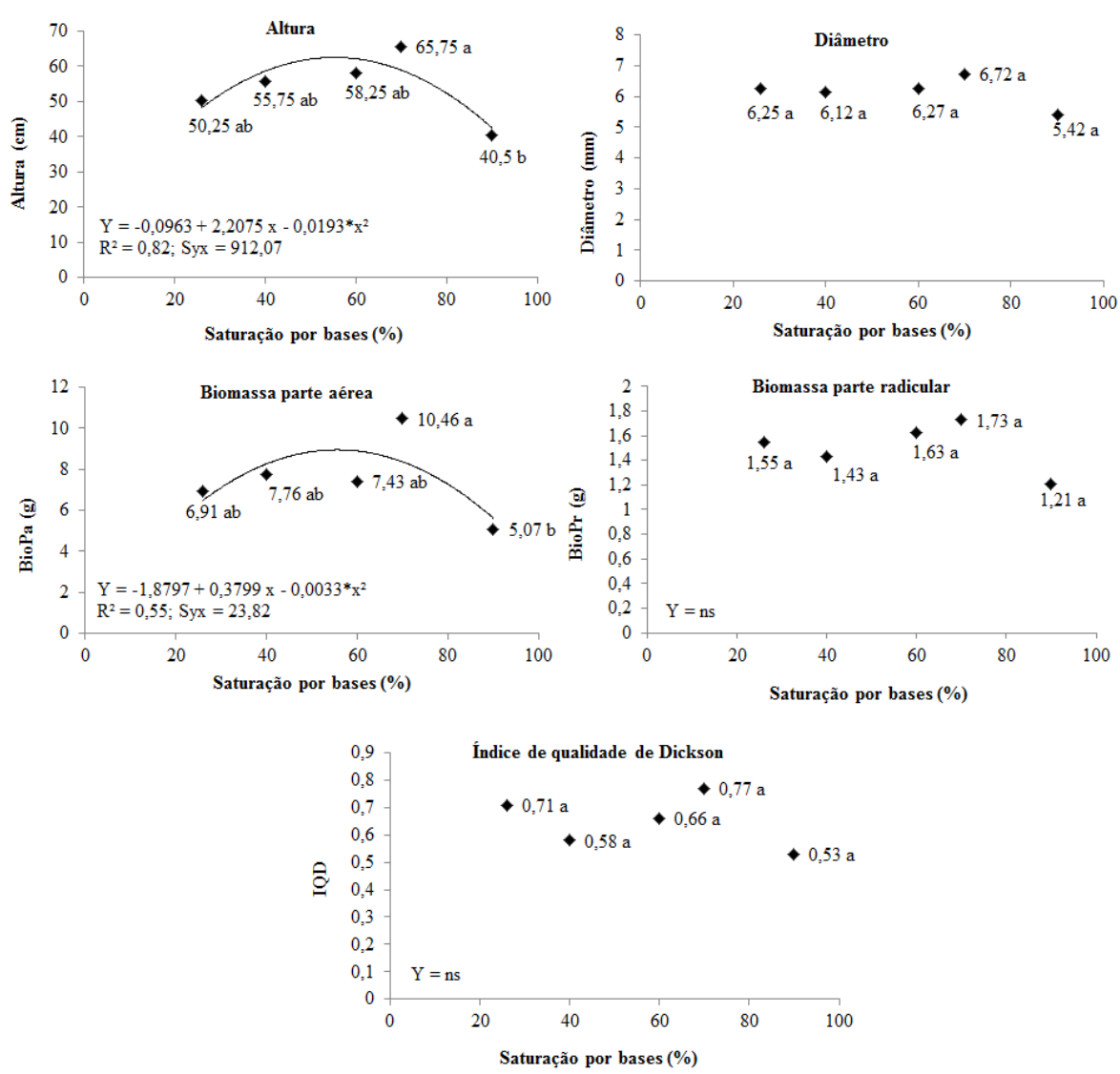

Figura 1. Equações de regressão para altura, diâmetro, biomassa da parte aérea, biomassa da parte radicular e índice de qualidade de Dickson de mudas de Schizolobium amazonicum submetidas a 
elevações da saturação por bases, após 120 dias. GL: graus de liberdade dos tratamentos = 4; graus de liberdade do resíduo $=15$; graus de liberdade da regressão $=1$.

$\mathrm{O}$ crescimento em altura foi superior em $\mathrm{V}=70 \%$ e o modelo de regressão que mais se adequou foi o quadrático, com aumento até a saturação de $70 \%$ e posterior queda, que pode ter ocorrido por ter afetado a disponibilidade de nutrientes devido o aumento no $\mathrm{pH}$ do substrato. Porém, a MET para altura foi observada na dose de 57,2\% e a MEE em 57,7\%, indicando o melhor desempenho para essa variável, entre as doses de 60 e $70 \%$ de saturação por bases. Souza et al. (2008) e Costa Filho et al. (2013), também observaram maior crescimento em altura das mudas após a aplicação de calcário ao substrato.

O crescimento em diâmetro foi $19 \%$ inferior nas mudas submetidas à saturação de $90 \%$ em relação à de 70\%, indicando uma tendência de aumento condizente com o que foi observado para o crescimento em altura. Isso é importante porque em um viveiro de produção de mudas, altura e diâmetro são as características morfológicas mais facilmente determinadas para identificar aquelas que estão aptas ao plantio definitivo no campo.

Quando os crescimentos em altura e em diâmetro ocorrem conjuntamente, garantem a obtenção de mudas de melhor qualidade e que, provavelmente, terão maior capacidade de sobreviver no campo. Dessa forma, observou-se, em todos os tratamentos, que as mudas de S. amazonicum estariam aptas para o campo antes dos 120 dias, pois, segundo Gonçalves et al. (2000) a altura das mudas devem estar entre 20 e $35 \mathrm{~cm}$ e o diâmetro de colo entre 5 e $10 \mathrm{~mm}$ para serem plantadas. Resultados superiores aos observados por Caione et al. (2012) ao adubar o solo para produção de mudas de $S$. amazonicum, com médias entre 36 e $43 \mathrm{~cm}$ de altura e 3,9 e 4,8 mm de diâmetro.

Os valores para a biomassa da parte aérea foram superiores nas mudas submetidas à saturação por bases de 70\%, com MET em 57,6\% e MEE em 60,6\%, comprovando que as doses entre 60 e 70\% proporcionaram melhores condições para a produção de biomassa da parte aérea das mudas de $S$. amazonicum. Resultados semelhantes foram observados por Bernardino et al. (2005, 2007) em mudas de Anadenanthera macrocarpa (Benth.) Brenan, e Dalbergia nigra (Vell.) Allemão ex Benth., respectivamente. Maeda \& Bognola (2012) e Costa Filho et al. (2013) também ajustaram equação quadrática para a biomassa da parte aérea, em mudas de Eucalyptus dunnii Maiden e de Mimosa caesalpinifolia (Benth.). Segundo Maeda \& Bognola (2012), essa resposta das plantas pode se dar porque a calagem aumenta a eficiência de utilização do $P$ para a produção de matéria seca da parte aérea. Além disso, a calagem aumenta a solubilidade dos demais macronutrientes e melhora as condições de crescimento do sistema radicular, facilitando a absorção de nutrientes.

Quanto à biomassa da raiz, a saturação por bases de $70 \%$ foi 30\% superior em relação à saturação de 90\%, não proporcionando significância entre os tratamentos. Gomes et al. (2004) também não observaram resposta significativa dos níveis de saturação por bases (24 e 60\%) na biomassa radicular de A. colubrina. Souza et al. (2008) atribuem esses resultados aos teores iniciais de $\mathrm{Ca}$ e de $\mathrm{Mg}$ presentes no substrato que podem ter sido suficientes para suprir a necessidade das plantas, pelo menos durante a fase de mudas. O que também pode ter ocorrido no presente estudo.

Portanto, a saturação por bases pouco influenciou no crescimento e desenvolvimento iniciais das mudas de S. amazonicum, considerando os parâmetros morfológicos analisados. Porém, há indicativos de que, a melhoria das condições de fertilidade do solo possa promover a produção de mudas mais resistentes e acelerar o crescimento, possibilitando o transplante para o campo anteriormente aos 120 dias de estabelecimento, na saturação por bases de $70 \%$.

\section{Nutrição das mudas}

A elevação da saturação por bases influenciou nos teores de macro e de micronutrientes nas mudas de S. amazonicum (Entra Figuras 2 e 3). A saturação por bases aumentou os teores de $P, K, S$, Fe e Mn nas folhas das mudas da espécie. Porém, não houve diferença significativa entre os tratamentos para os teores de N, Ca e Zn. 

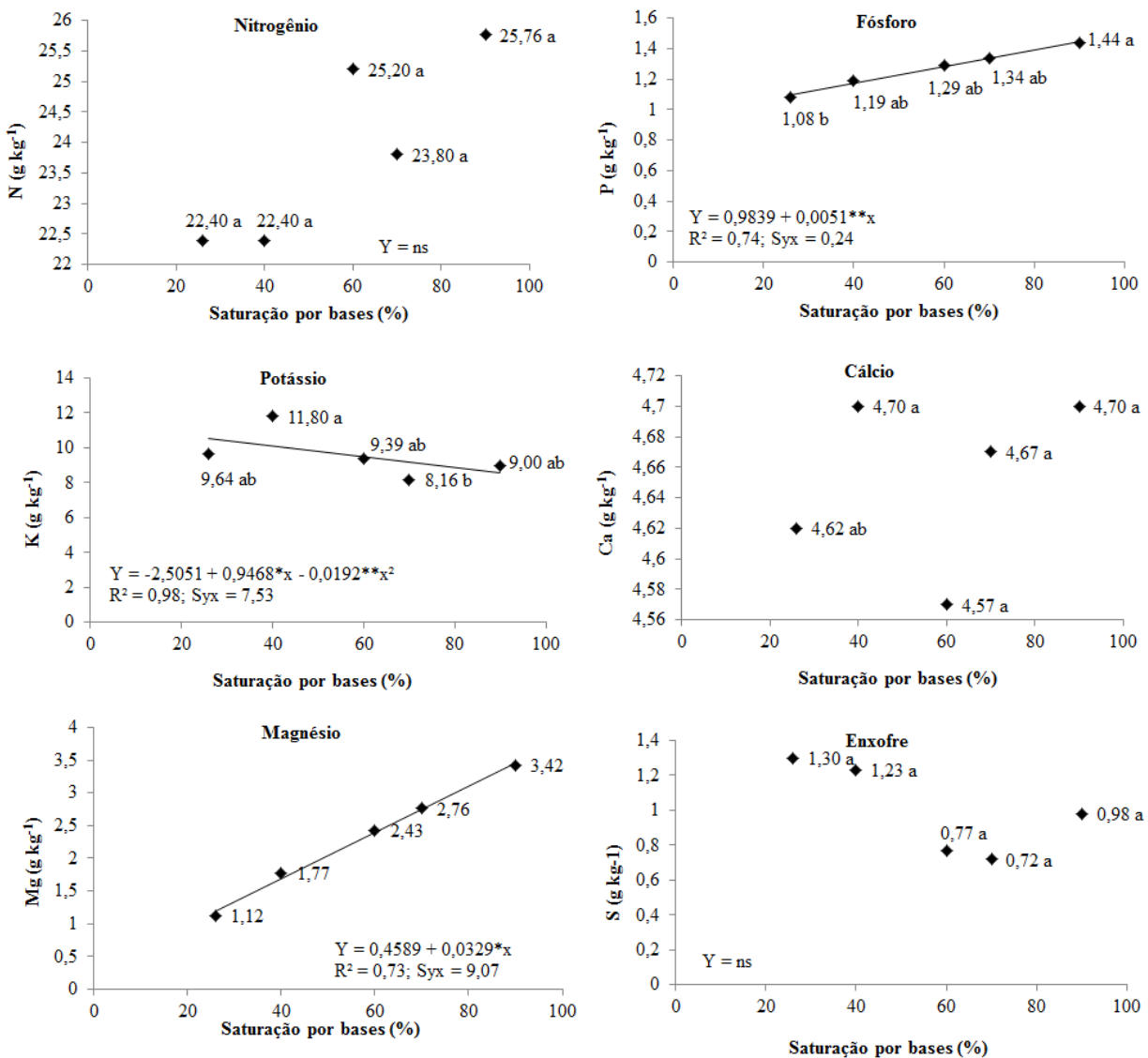

Figura 2. Teores de N, P, K, Ca, Mg e S em mudas de Schizolobium amazonicum submetidas a elevações da saturação por bases, após 120 dias. GL: graus de liberdade dos tratamentos = 4; graus de liberdade do resíduo = 15; graus de liberdade da regressão $=1$.

Os teores de $\mathrm{N}$ (Figura 2) nas mudas de S. amazonicum não atingiram valores significativamente superiores às do tratamento testemunha, porém, houve tendência de aumento com a elevação da saturação por bases. Além disso, todos os tratamentos proporcionaram teores considerados adequados segundo recomendação de Malavolta et al. (1997). Provavelmente como consequência da aplicação de calcário, que foi eficiente na mineralização do $\mathrm{N}$, melhorando o pH do solo, disponibilizando o nutriente para a planta. Marques et al. (2004a, 2004b) e Caione et al. (2012) também atestaram as exigências em N pelas mudas de $S$. amazonicum. Isso porque, na sua ausência, as plantas dessa espécie podem apresentar redução na síntese de clorofila nas folhas mais velhas (Marques et al., 2004b). Provavelmente, porque $\mathrm{N}$ participa da absorção iônica, fotossíntese, respiração, multiplicação e da diferenciação celular (Marschner, 1995). Com a planta bem suprida, o crescimento ocorre adequadamente, o que pôde ser observado para a espécie estudada.

Os teores de P (Figura 2) foram crescentes conforme a elevação da saturação por bases, ou seja, foram influenciados pela saturação por bases, obtendo os maiores valores na saturação de $90 \%$ e equação linear. Porém, a MET para os teores de P foi obtida em $96 \%$ de saturação e a MEE em $98 \%$, indicando que essa eficiência estaria em um nível superior ao que foi testado no presente estudo. Todos os tratamentos com teores adequados segundo recomendação de Malavolta et al. (1997), de 1,0 a 2,3 $\mathrm{g} \mathrm{kg}^{-1}$. Esses teores de $\mathrm{P}$ estão relacionados diretamente com o aumento do pH do solo, segundo Fernandes et al. (2003), a calagem eleva os valores de $\mathrm{pH}$ do solo, reduz a atividade de $\mathrm{Al}$ e $\mathrm{Fe}$ e aumenta a disponibilidade de nutrientes, notadamente do P. O que também influenciou no crescimento das mudas de S. amazonicum. De acordo Gomes \& Paiva (2004) um adequado suprimento em $\mathrm{P}$ é importante para a formação dos primórdios vegetativos. Além disso, a geometria das raízes influencia o crescimento da planta e a aquisição de nutrientes, especialmente daqueles com baixa mobilidade no solo, a exemplo do P (Stahl et al., 2013) e do K, o que depende 
diretamente do desenvolvimento radicular (Matos et al., 2012). O que pode ter facilitado o desenvolvimento radicular das mudas, propiciando a absorção dos nutrientes.

A elevação da saturação por bases reduziu os teores de K (Figura 2). Segundo Marschner (1990) o aumento de $\mathrm{pH}$ reduz o estímulo na absorção de K, causado pelo Ca, podendo ocorrer até inibição da absorção de K, provavelmente devido à competição. Somente na saturação de $40 \%$ os teores foram considerados adequados, segundo a recomendação de Malavolta et al. (1997) de 10 a $15 \mathrm{~g} \mathrm{~kg}^{-1}$. Nesse caso, a MET foi obtida na dose de 24,7\% e a MEE em 25,2\%, indicando que a menor dose (testemunha) foi suficiente para manter os teores de $\mathrm{K}$ para a nutrição das mudas de S. amazonicum. Teores adequados de K são importantes porque esse elemento faz parte da abertura e fechamento dos estômatos e, portanto, está diretamente ligado à taxa de transpiração (Favare et al., 2012). No entanto, os resultados obtidos no presente experimento não levaram ao aparecimento de sintomas de deficiências nas mudas, pois, de acordo om Marques et al. (2004b) na ausência de K, as mudas de S. amazonicum apresentam menor crescimento e menor número de folhas. Portanto, os resultados obtidos nesse experimento podem ter ocorrido devido a pouca exigência da espécie pelo elemento, também constatado por Caione et al. (2012).

Os teores de Ca e de Mg (Figura 2) foram influenciadas pelo aumento da saturação por bases, com valores ideais, entre 3,0 a 12,0 $\mathrm{g} \mathrm{kg}^{-1}$, segundo Malavolta et al. (1997). Condições que podem ser atribuídas à adição de Ca, porém, no tratamento testemunha, as plantas podem ter absorvido o nutriente de suas reservas. O que permitiu não haver significância entre os tratamentos. Resultados estes, semelhantes aos de Sena et al. (2010) em Dinizia excelsa Ducke. Isso pode ser explicado pela necessidade que o S. amazonicum possui de Ca, superior ao de Mg, constatado por Marques et al. (2004a). De acordo com Marques et al. (2004a) mudas dessa espécie, com deficiência de Ca apresentam menor número de folhas, queda prematura dos folíolos e murcha da gema apical. As raízes mostram-se menos desenvolvidas e mais espessas com poucas raízes laterais. Enquanto que, a saturação por bases elevou os teores de Mg, que se destacaram quando submetidas a $90 \%$ e; os menores foram observados no tratamento sem aplicação de calcário, porém, dentro da faixa ideal recomendada por Malavolta et al. (1997), entre 1,5 e 5,0 g kg-1. O método utilizado para as demais características não foi eficiente em calcular MET e MEE para os teores de $\mathrm{Mg}$, provavelmente devido à equação ajustada, nesse caso, ser linear.

O tratamento testemunha foi o que disponibilizou a maior média para os teores de $S$ (Figura 2) para as mudas de $S$. amazonicum, possibilitando teores adequados de $\mathrm{S}$, segundo recomendação de Malavolta et al. (1997). Portanto, a saturação por base influenciou negativamente na disponibilidade de S, com os menores teores na saturação de 70\%. Porém, sem aparecimento de sintomas de deficiências. Marques et al. (2004b) observaram, na ausência de $S$, mudas com folhas novas cloróticas, sem redução no tamanho da planta ou no número de folhas. O que não foi observado no presente caso.

Os teores de Fe (Figura 3) foram alterados com o aumento da saturação por bases até $90 \%$, tendo sido esta a que mais limitou a disponibilidade de Fe nas folhas das mudas de S. amazonicum. Nesse caso, a MET foi obtida na dose de 49,2\% e a MEE em 49,5\%, indicando que as doses entre 40 e $60 \%$ foram mais favoráveis para os teores de Fe nas mudas da espécie. Segundo Malavolta et al. (1997), esses teores devem estar entre 25 e $200 \mathrm{mg} \mathrm{kg}^{-1}$, porém, foram observados valores superiores em todos os tratamentos testados, sem que isso tenha afetado o crescimento das mudas. Marques et al. (2004a, 2004b) também observaram que Fe foi um dos micronutrientes mais absorvidos pelas mudas de S. amazonicum. Sendo que, a ausência de Fe reduz drasticamente o crescimento das mudas dessa espécie (Marques et al., 2004b). O que ocorre porque o Fe é essencial no metabolismo energético, na fixação do $\mathrm{N}$ e no desenvolvimento do caule e raízes (Taiz \& Zeiger, 2004). 

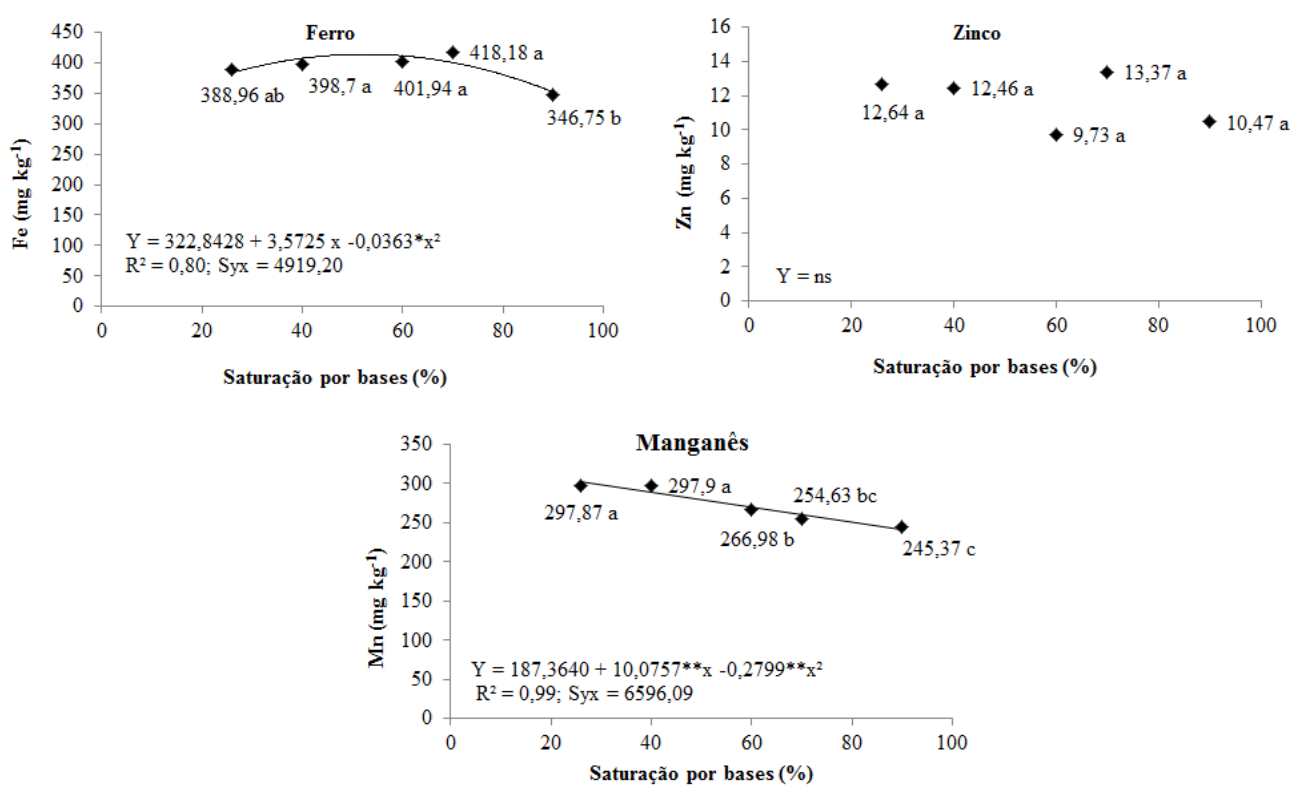

Figura 3. Teores de Fe, Mn e Zn em mudas de Schizolobium amazonicum submetidas a elevações da saturação por bases, após 120 dias. GL: graus de liberdade dos tratamentos = 4; graus de liberdade do resíduo $=15 ;$ graus de liberdade da regressão $=1$.

Os teores de Mn (Figura 3) foram favorecidos pelas saturações natural e de $40 \%$. Ao calcular a MET verificou-se que essa se apresentou fora das doses testadas no estudo, que foi de $18 \%$, assim como a MEE, indicando que o solo já poderia ter teores de Mn suficientes para o S. amazonicum. Isso pode ser constatado ao se observar a Figura 3, já que os teores de Mn já se apresentam superiores a $290 \mathrm{mg} \mathrm{kg}^{-1}$ no tratamento testemunha. Seguindo recomendação de Martinez et al. (1999), na qual os teores de Mn devem estar na faixa de 100 a $600 \mathrm{mg} \mathrm{kg}^{-1}$, verificou-se que, estiveram dentro da faixa ideal em todos os tratamentos testados. O que é importante porque o Mn participa do metabolismo energético respiratório (Taiz \& Zeiger, 2004), compreendendo-se, dessa forma, porque promove o crescimento e desenvolvimento das plantas. No entanto, a calagem parece ter influenciado negativamente na disponibilidade de Mn. Segundo Chimello (2001); Consolini \& Coutinho (2004), a diminuição na disponibilidade de $\mathrm{Mn}$ no solo, devido ao aumento do $\mathrm{pH}$, pode ser atribuída à redistribuição desses cátions do reservatório trocável para os reservatórios óxidos de ferro e alumínio cristalino e pouco cristalino. No entanto, Marques et al. (2004b) não observaram redução no crescimento de $S$. amazonicum na ausência de Mn.

Os teores de Zn (Figura 3) não foram alterados pela saturação por bases, não havendo significância entre os tratamentos. Se considerar a recomendação de Malavolta (1980), de $20 \mathrm{mg} \mathrm{kg}^{-1}$, nenhum tratamento se enquadrou na faixa. Segundo Malavolta et al. (1997), à medida em que aumenta o $\mathrm{pH}$ do meio, a disponibilidade de $\mathrm{Zn}$ é reduzida linearmente, o que não foi observado nas mudas de $S$. amazonicum, provavelmente, devido as exigências da espécie e a fase de crescimento. Resultados semelhantes aos observados por Sena et al. (2010).

Portanto, a elevação na saturação por bases pode influenciar na concentração de nutrientes, aumentando ou diminuindo sua disponibilidade e/ou absorção pelas mudas de $S$. amazonicum, podendo acarretar em deficiência ou toxidez de alguns nutrientes. Sendo assim, deve-se atentar para as exigências da espécie, pois ela adapta às condições nutricionais variadas, desde $20 \%$ a $70 \%$ de saturação por bases. No entanto, na saturação por bases de $40 \%$ já se obtém concentrações adequadas dos nutrientes para a planta. 


\section{CONCLUSÃO}

O crescimento das mudas de S. amazonicum foi pouco influenciado pela elevação na saturação por bases do substrato, até os 120 dias de estabelecimento. No entanto, obtemse maior produção de biomassa na parte aérea na saturação de $70 \%$.

As concentrações de macro e de micronutrientes foram alteradas pela saturação por bases, cujas concentrações mais adequadas variaram conforme o nutriente analisado.

Os cálculos de MET e de MEE indicam que os melhores resultados foram obtidos para as saturações por bases entre 55 e $60 \%$, considerando as variáveis de crescimento e de nutrição, testadas no presente estudo.

Outros estudos devem ser realizados para estudar os efeitos de saturações entre 50 e $70 \%$, a fim de verificar se existe uma resposta mais correta, com base nas variáveis morfológicas e nutricionais e que possam ser compatíveis com a MET e MEE.

\section{REFERÊNCIAS BIBLIOGRÁFICAS}

Almeida, D. H., Scaliante, R. M., Macedo, L. B., Macêdo, A. N., Dias, A. A., Christoforo, A. L., \& Calil Junior, C. (2013). Caracterização completa da madeira da espécie amazônica paricá (Schizolobium amazonicum Herb.) em peças de dimensões estruturais. Revista Árvore, 37(6), 1175-1181. http://dx.doi.org/10.1590/S0100-67622013000600019.

Alvarez, V. H. (1974). Equilíbrio de formas disponíveis de fósforo e enxofre em dois latossolos de Minas Gerais (Dissertação de mestrado). Faculdade de Agronomia, Universidade Federal de Viçosa, Viçosa.

Bernardino, D. C. S., Paiva, H. N., Neves, J. C. L., Gomes, J. M., \& Marques, V. B. (2005). Crescimento e qualidade de mudas de Anadenanthera macrocarpa (Benth.) Brenan em resposta à saturação por bases do substrato. Revista Árvore, 29(6), 863-870. http://dx.doi.org/10.1590/S010067622005000600004.

Bernardino, D. C. S., Paiva, H. N., Neves, J. C. L., Gomes, J. M., \& Marques, V. B. (2007). Influência da saturação por bases e da relação Ca:Mg do substrato sobre o crescimento inicial de jacarandá-dabahia (Dalbergia nigra (Vell.) Fr. All.Ex Benth.). Revista Árvore, 31(4), 567-573. http://dx.doi.org/10.1590/S0100-67622007000400001.

Braga, M. M., Furtini Neto, A. E., \& Oliveira, A. H. (2015). Influência da saturação por bases na qualidade e crescimento de mudas de cedro-australiano (Toona ciliata M. Roem var. australis). Ciência Florestal, 25(1), 49-58. http://dx.doi.org/10.5902/1980509817462.

Caione, G., Lange, A., \& Schoninger, E. L. (2012). Crescimento de mudas de Schizolobium amazonicum (Huber ex Ducke) em substrato fertilizado com nitrogênio, fósforo e potássio. Scientia Forestalis, 40(94), 213-221.

Cardoso, A. A. S., Santos, J. Z. L., Tucci, C. A. F., Farias, E. P., \& Moura, R. P. M. (2015). Influência da acidez e do teor de fósforo do solo no crescimento inicial do mogno. Pesquisa Florestal Brasileira, 35(1), 1 10. http://dx.doi.org/10.4336/2015.pfb.35.81.667.

Chimello, M. A. (2001). Efeito do pH do solo e da aplicação de manganês na distribuição e disponibilidade do micronutriente para a soja (Glycine max. (L.) Merrill) (Dissertação de mestrado). Faculdade de Ciências Agrárias e Veterinárias, Universidade Estadual Paulista, Jaboticabal.

Colli, A., Vital, B. R., Carneiro, A. C. O., Silva, J. C., Carvalho, A. M. M. L., \& Lucia, R. M. D. (2010). Propriedades de chapas fabricadas com partículas de madeira de paricá (Schizolobium amazonicum Huber ex. Ducke) e fibras de coco (Coco nucifera L.). Revista Árvore, 34(2), 333-338. http://dx.doi.org/10.1590/S0100-67622010000200016.

Consolini, F., \& Coutinho, E. L. M. (2004). Efeito da aplicação de Zn e do pH do solo na disponibilidade do micronutriente. Acta Scientiarum. Agronomy, 26(1), 7-12. http://dx.doi.org/10.4025/actasciagron.v26i1.1948.

Costa Filho, R. T., Valeri, S. V., \& Cruz, M. C. P. (2013). Calagem e adubação fosfatada no crescimento de mudas de Mimosa caesalpinifolia Benth. em Latossolo vermelho-amarelo. Ciência Florestal, 23(1), 8998. http://dx.doi.org/10.5902/198050988442.

Cruz, C. A. F., Paiva, H. N., Gomes, K. C. O., \& Guerrero, C. R. A. (2004). Efeito de diferentes níveis de saturação por bases no desenvolvimento e qualidade de mudas de ipê-roxo (Tabebuia impetiginosa (Mart.) Standley). Scientia Forestalis, 66, 100-107.

Dickson, A., Leaf, A. L., \& Hosner, J. F. (1960). Quality appraisal of white spruce and white pine seedlings stock in nurseries. Forestry Chronicle, 36(1), 10-13. http://dx.doi.org/10.5558/tfc36010-1. 
Empresa Brasileira de Pesquisa Agropecuária - EMBRAPA, \& Centro Nacional de Pesquisa do Solo. (1997). Manual de métodos de análise do solo (2. ed., 212 p.). Brasília: EMBRAPA.

Empresa Brasileira de Pesquisa Agropecuária - EMBRAPA, \& Centro Nacional de Pesquisa do Solo. (2006). Sistema brasileiro de classificação de solos (2. ed., 412 p.). Brasília: EMBRAPA.

Favare, L. G., Guerrini, I. A., \& Backes, C. (2012). Níveis crescentes de saturação por bases e desenvolvimento inicial de teca em um Latossolo de textura média. Ciência Florestal, 22(4), 693-702. http://dx.doi.org/10.5902/198050987551.

Fernandes, A. R., \& Linhares, L. C. F., Morais, F. I. O., \& Silva, G. R. (2003). Características químicas do solo, matéria seca e acumulação de minerais nas raízes de adubos verdes, em resposta ao calcário e ao fósforo. Revista de Ciências Agrárias (Belém), 40, 45-54.

Freitas, E. C. S., Paiva, H. N., Leite, H. G., \& Oliveira Neto, S. N. (2017). Efeito da adubação fosfatada e saturação por bases do substrato sob o crescimento e a qualidade de mudas de Plathymenia foliolosa Benth. Revista Árvore, 41(1), 1-9.

Gazel Filho, A. B., Cordeiro, I. M. C. C., Alvarado, J. R., \& Santos Filho, B. G. (2007). Produção de biomassa em quatro procedências de paricá (Schizolobium parahyba var. amazonicum (Huber ex Ducke)) Barneby no estádio de muda. Revista Brasileira de Biociências, 5(2), 1047-1049.

Gomes, J. M., \& Paiva, H. (2004). Viveiros florestais: propagação sexuada (116 p.). Viçosa: UFV.

Gomes, K. C. O., Paiva, H. N., Neves, J. C. L., Barros, N. F., \& Silva, S. R. (2004). Influência da saturação por bases e do fósforo no crescimento de muda de angico-branco. Revista Árvore, 28(6), 785-792. http://dx.doi.org/10.1590/S0100-67622004000600003.

Gonçalves, J. L. M., Santarelli, E. G., Moraes Neto, S. P., \& Manara, M. P. (2000). Produção de mudas de espécies nativas: substrato, nutrição, sombreamento e fertilização. In J. L. M. Gonçalves \& V. Benedetti. Nutrição e fertilização florestal (pp. 310-350). Piracicaba: IPEF.

Iwakiri, S., Zeller, F., Pinto, J. A., Ramirez, M. G. L., Souza, M. M., \& Seixas, R. (2010). Avaliação do potencial de utilização da madeira de Schizolobium amazonicum "paricá" e Cecropia hololeuca "embaúba" para produção de painéis aglomerados. Acta Amazonica, 40(2), 303-308. http://dx.doi.org/10.1590/S0044-59672010000200008.

Maeda, S., \& Bognola, I. A. (2012). Influência de calagem e adubação fosfatada no crescimento inicial de eucalipto e nos níveis críticos de P. Pesquisa Florestal Brasileira, 32(72), 401-407. http://dx.doi.org/10.4336/2012.pfb.32.72.401.

Malavolta, E. (1980). Elementos de nutrição mineral de plantas (251 p.). São Paulo: Ceres.

Malavolta, E., Vitti, G. C., \& Oliveira, S. A. (1997). Avaliação do estado nutricional das plantas: princípios e aplicações (2. ed., 319 p.). Piracicaba: Potafos.

Marques, T. C. L. L. S. M., Carvalho, J. G., Lacerda, M. P. C., \& Mota, P. E. F. (2004a). Exigências nutricionais do paricá (Schizolobium amazonicum Herb.) na fase de muda. Cerne, 10(2), 167-183.

Marques, T. C. L. L. S. M., Carvalho, J. G., Lacerda, M. P. C., \& Mota, P. E. F. (2004b). Crescimento inicial do paricá (Schizolobium amazonicum) sob omissão de nutrientes e de sódio em solução nutritiva. Cerne, 10(2), 184-195.

Marschner, H. (1990). Mineral nutrition of higher plants (674 p.). London: Academic.

Marschner, H. (1995). Mineral Nutrition of higler plants (2. ed., 889 p.). New York: Academic Press.

Martinez, H. E. P., Carvalho, J. G., \& Souza, R. B. (1999). Diagnose foliar. In A. C. Ribeiro, P. T. G. Guimarães, \& V. H. Alvares. Recomendações para o uso de corretivos e fertilizantes em Minas Gerais (5. ed., p. 143-167). Viçosa: CFSEMG/UFV.

Matos, G. S. B., Silva, G. R., Gama, M. A. P., Vale, R. S., \& Rocha, J. E. C. (2012). Desenvolvimento inicial e estado nutricional de clones de eucalipto no nordeste do Pará. Acta Amazonica, 42(4), 491-500. http://dx.doi.org/10.1590/S0044-59672012000400006.

Oliveira, J. R., Souza, F. V. P., Silva, U. T. G., Duarte, N. F., \& Pinto, S. I. C. (2015). Saturação por bases para o cultivo do cedro australiano. Global Science and Technology, 8(2), 96-102. http://dx.doi.org/10.14688/1984-3801/gst.v8n2p96-102.

Passos, M. A. A. (1994). Efeito da calagem e de fósforo no crescimento inicial da algaroba (Prosopis juliflora (SW) DC) (Tese de doutorado). Departamento de Fitotecnia, Universidade Federal de Viçosa, Viçosa.

Rosa, L. S., Vieira, T. A., Santos, D. S., \& Silva, L. C. B. (2009). Emergência, crescimento e padrão de qualidade de mudas de Schizolobium amazonicum Huber ex Ducke sob diferentes níveis de sombreamento e profundidades de semeadura. Revista de Ciências Agrárias (Belém), 52, 87-98. 
Sena, J. S., Tucci, C. A. F., Lima, H. N., \& Hara, F. A. S. (2010). Efeito da calagem e da correção dos teores de Ca e Mg do solo sobre o crescimento de mudas de angelim-pedra (Dinizia excelsa Ducke). Acta Amazonica, 40(2), 309-318. http://dx.doi.org/10.1590/S0044-59672010000200009.

Souza, P. H., Paiva, H. N., Neves, J. C. L., Gomes, J. M., \& Marques, L. S. (2008). Influência da saturação por bases do substrato no crescimento e qualidade de mudas de Machaerium nictitans (Vell.) Benth. Revista Árvore, 32(2), 193-201. http://dx.doi.org/10.1590/S0100-67622008000200001.

Stahl, J., Ernani, P. R., Gatiboni, L. C., Chaves, D. M., \& Neves, C. U. (2013). Produção de massa seca e eficiência nutricional de clones de Eucalyptus dunnii e Eucalyptus benthamii em função da adição de doses de fósforo ao solo. Ciência Florestal, 23(2), 287-295. http://dx.doi.org/10.5902/198050989275.

Stork, L., Garcia, D. C., \& Lopes, S. J. (2000). Experimentação vegetal (198 p.). Santa Maria: UFSM.

Taiz, L., \& Zeiger, E. (2004). Fisiologia vegetal (3. ed., 719 p.). Porto Alegre: Artmed.

Vargas, G., \& Marques, R. (2017). Crescimento e nutrição de angico e canafístula sob calagem e gessagem. Floresta e Ambiente, 24, 1-10. http://dx.doi.org/10.1590/2179-8087.010216.

Vidaurre, G. B., Carneiro, A. C. O., Vital, B. R., Santos, R. C., \& Valle, M. L. A. (2012). Propriedades energéticas da madeira e do carvão de paricá (Schizolobium amazonicum). Revista Árvore, 36(2), 365371. http://dx.doi.org/10.1590/S0100-67622012000200018.

Contribuição dos Autores: CRV: Análise formal, investigação, administração do projeto, supervisão, escrita primeira redação, escrita - revisão e edição; OLSW: Conceituação, curadoria de dados, metodologia, supervisão, validação; JFS: Conceituação, curadoria de dados, metodologia, supervisão, validação. 